\title{
Analysis of miRNA expression profiling identifies miR-214-3p as a novel biomarker in sinonasal inverted papilloma
}

\author{
Yaoshu Teng ${ }^{\star \star \ddagger}, 1,2$, Yuandong $\mathrm{Li}^{\ddagger}, 2$, Zhihong Lin ${ }^{\ddagger}, 3$, Yueqiu Gao ${ }^{1,2}$, Xiaolin Cao ${ }^{1,2}$, Xiangyu \\ Lou $^{1,2}$, Fengchun $\operatorname{Lin}^{4} \&$ Yong Li ${ }^{1,2}$ \\ ${ }^{1}$ Department of Otorhinolaryngology, Affiliated Hangzhou First People's Hospital, Zhejiang University School of Medicine, 310006 \\ Hangzhou, PR China \\ ${ }^{2}$ Department of Otorhinolaryngology, The Fourth Clinical Medical College, Zhejiang Chinese Medical University, 310006 \\ Hangzhou, PR China \\ ${ }^{3}$ Department of Otorhinolaryngology, Second Affiliated Hospital, School of Medicine, Zhejiang University, 310008 Hangzhou, PR \\ China \\ ${ }^{4}$ Department of Pathology, Second Affiliated Hospital, School of Medicine, Zhejiang University, 310008 Hangzhou, PR China \\ *Author for correspondence: tengyaoshu@163.com \\ $\ddagger$ Authors contributed equally
}

Aim: To analyze the expression profile, diagnostic and clinicopathological significances of miRNAs in sinonasal inverted papilloma (SNIP). Materials \& methods: The expression profile of miRNAs was analyzed using a miRNA microarray approach. The potential functions and clinical significances of specific miRNAs were further analyzed by bioinformatics and statistical methods. Results: The microarray assay identified 37 significantly upregulated and 21 downregulated miRNAs in SNIP. Of nine miRNAs randomly selected, the expression levels of seven miRNAs were confirmed by quantitative real-time PCR. The potential target genes of several candidate miRNAs were enriched in some biological processes and cellular signaling pathways related to tumorigenesis. Receiever operating characteristic curve analysis for miR-214-3p indicated an area under the curve of 0.932 . Notably, its expression level was significantly decreased in SNIP tissues and associated with SNIP staging and recurrence. Conclusion: MiR-214-3p can possibly serve as a valuable biomarker and a therapeutic target for SNIP.

\section{Graphical abstract:}

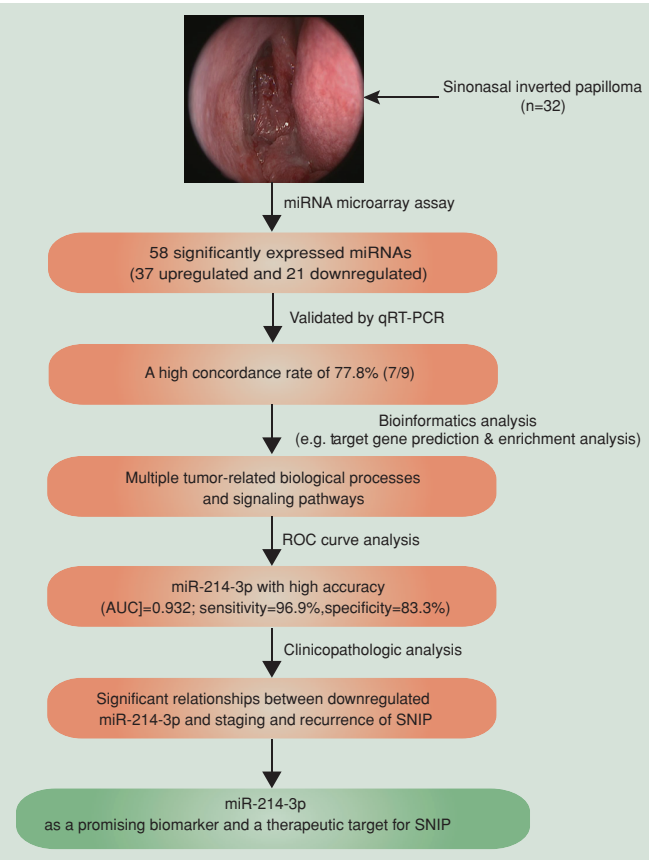


First draft submitted: 13 May 2018; Accepted for publication: 8 June 2018; Published online: 28 June 2018

Keywords: bioinformatics $\bullet$ biomarker $\bullet$ Human papilloma virus $\bullet$ inverted papilloma $\bullet$ microarray $\bullet$ microRNA $\bullet$ pathogenesis $\bullet$ pathway $\bullet$ sinonasal tumors $\bullet$ target genes

Sinonasal inverted papilloma (SNIP) is a benign epithelial neoplasm arising from the Schneiderian mucosa of nasal cavity and paranasal sinuses, accounting for $0.5-4 \%$ of all primary nasal tumors [1]. The highest incidence of SNIP is observed in the fifth and sixth decades of life, with the male to female ratio of 2-5:1 [2,3]. Although SNIP is a histologically benign lesion, it is characterized by local invasiveness, a high recurrence rate and a tendency for malignant transformation [4]. The exact etiologies of SNIP remain to be explored. Human papilloma virus (HPV) infection is generally recognized to be involved in the progression of SNIP. Other possible etiologies include allergy, chronic inflammation, smoking and occupational exposure [5]. However, little is known about the underlying molecular genetic alterations and physiopathologic mechanism of this clinical entity.

MiRNAs are a class of small (approximately 22 nucleotides), single-stranded noncoding RNAs that negatively regulate post-transcriptional protein-coding gene expression through specifically binding to $3^{\prime}$ untranslated region of target mRNAs, leading to mRNA degradation or translation inhibition [6,7]. MiRNAs play important roles in various physiological and pathological processes, including individual development, tumorigenesis, immune responses and so on [8-10]. Accumulating evidence indicates that some miRNAs affect the expression of genes and signaling pathways involved in the pathogenesis of head and neck tumors [11-13]. Kakizaki et al. [14] found that a total of 200 miRNAs exhibited a greater than twofold differential expression between SNIP and squamous cell carcinoma (SCC) arising from SNIP. Interestingly, miR-296-3p was thought to play a critical role in the malignant transformation of SNIP through the regulation of PTEN. However, genome-wide profiling, diagnostic and clinicopathological significances of miRNAs in SNIP appear to have received little attention.

In this study, to further elucidate the potential functions and clinical value of specific miRNAs, we characterized the miRNA expression profile of SNIP tissue with microarray analysis to distinguish the significantly upregulated and downregulated miRNAs. Based on the microarray data, we predicted the potential functions of the selected miRNAs and explored the associations between the altered miRNA expression and clinicopathological characteristics of SNIP by bioinformatics and statistical analysis. Together, our data lay the foundation for future functional and mechanism studies of SNIP-related miRNAs, and may provide novel targets for therapeutic intervention against SNIP.

\section{Materials \& methods}

Patients \& sample collection

SNIP tissue samples were obtained surgically from 32 patients with SNIP (26 males and six females; mean age 53.7 years; range: $32-85$ years as the SNIP group). The diagnosis of SNIP was confirmed by histopathological examination. Detailed characteristics of these patients were summarized in Table 1. A total of 12 patients with only nasal septum deviation were selected to provide nasal mucosal tissue samples (nine males and three females; mean age 51.2 years; range: $28-64$ years as the control group). Patients were admitted to the Departments of Otorhinolaryngology in Hangzhou First People's Hospital, Nanjing Medical University and Second Affiliated Hospital, School of Medicine, Zhejiang University between 2012 and 2016. All tissue samples with clinical data were immediately preserved in RNAlater Solution (Ambion, TX, USA) after resection and then stored at $-20^{\circ} \mathrm{C}$ until use. This study complied with the Declaration of Helsinki and was approved by the local ethics committee. Written informed consent was obtained at enrollment from each participating subject.

\section{RNA extraction}

Total RNA was extracted from the tissue samples and purified using mirVana ${ }^{T M}$ miRNA Isolation Kit without phenol (Ambion, TX, USA) following the manufacturer's instructions. The RNA concentration was quantified using a NanoDrop spectrophotometer (Thermo Fisher Scientific, MA, USA). The integrity and quality of RNA was assessed by an Agilent Bioanalyzer 2100 (Agilent Technologies, CA, USA), and the RNA samples with an RNA integrity number $\geq 6.0$ and $28 \mathrm{~S} / 18 \mathrm{~S}>0.7$ were deemed acceptable to perform the miRNA microarray assay and reverse transcription. 


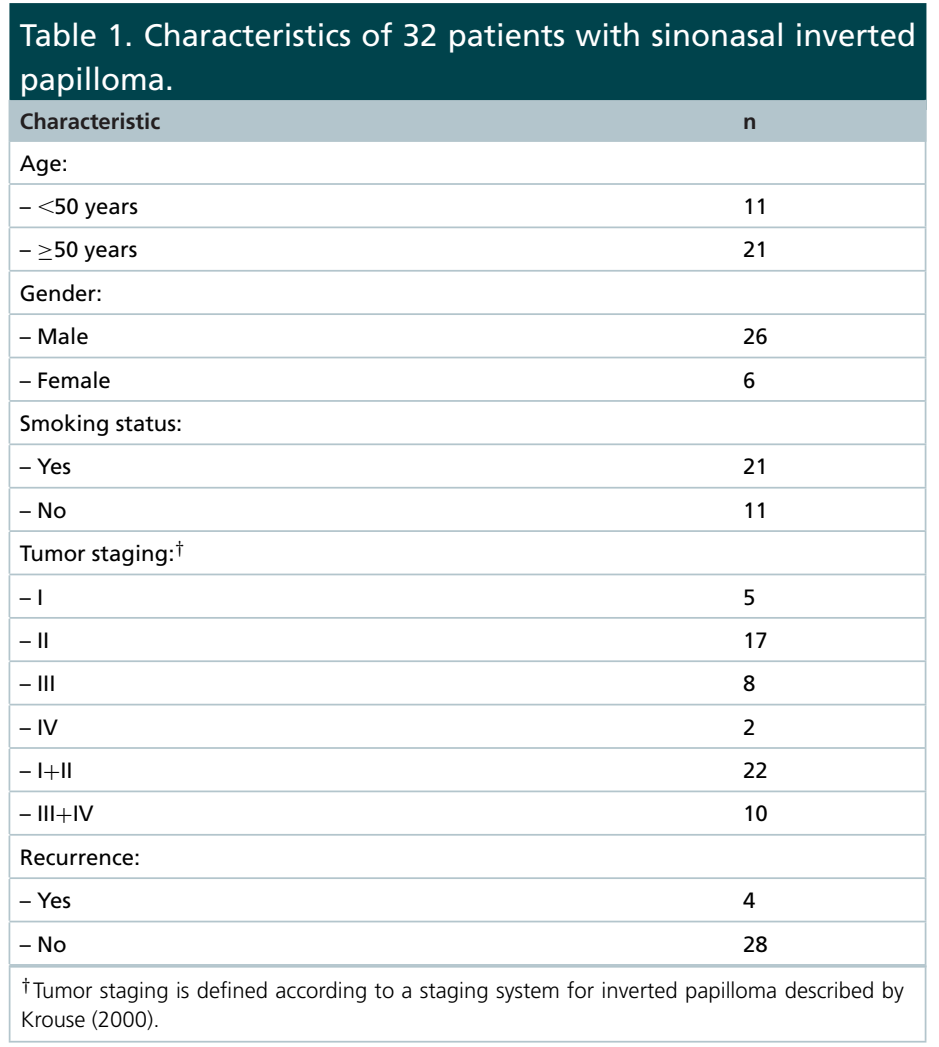

\section{MiRNA microarray assay}

The RNA samples were analyzed at Shanghai Biotechnology Corporation (Shanghai, China) using the Agilent Human miRNA Microarray $(8 \times 60 \mathrm{~K}$; version 21.0) with capture probes for a total of 2549 human miRNAs based on the Sanger miRBase database (release 21.0). Microarray assay was performed according to the manufacturer's instructions. Briefly, the miRNA molecules in total RNA were labeled using miRNA Complete Labeling and Hyb Kit (Agilent Technologies). Each microarray slide was hybridized using $100 \mathrm{ng}$ Cy3-labeled RNA in a hybridization oven (Agilent technologies, CA, USA). After hybridization, the slides were washed using Gene Expression Wash Buffer Kit (Agilent technologies), and then scanned using the Agilent Microarray Scanner (Agilent Technologies) and Feature Extraction software program (version 10.7, Agilent technologies) with default settings. Raw data were normalized by Quantile algorithm, Gene Spring software program (version 12.6, Agilent technologies). A relative fold change $>3$ in the differential expression of miRNAs and a p-value $<0.01$ were considered significant. The Gene Cluster (version 3.0) and Java Tree View software programs were used to perform the hierarchical cluster analysis of differentially expressed miRNAs and to visualize the miRNAs in the form of a heat map.

\section{Quantitative real-time reverse transcription PCR}

A cDNA synthesis was carried out using miScript II reverse transcription Kit (Qiagen, CA, USA). To validate the quantity of miRNA, quantitative real-time reverse transcription PCR (qRT-PCR) was performed on $7900 \mathrm{HT}$ Sequence Detection System (ABI, CA, USA) with miRcute miRNA qPCR Detection Kit (SYBR Green, Tiangen, China) according to the manufacturer's protocol. Forward primers specific to the mature miRNA from the Sanger miRNA database (Table 2) were synthesized by Invitrogen (Shanghai, China). Expression analysis was carried out in triplicate for each sample. The endogenous control for normalization was U6 small RNA, and the expression levels of miRNA were calculated using the formula $2^{-\Delta \mathrm{CT}}\left(\Delta \mathrm{Ct}=\mathrm{Ct}_{\mathrm{miRNA}}-\mathrm{Ct}_{\mathrm{U}}\right)$.

\section{Enrichment analysis \& network construction}

The target genes for the significantly differentially expressed miRNAs were predicted using different algorithms, including TargetScan Human Release 7.1 (www.targetscan.org/), miRanda (www.microrna.org/) and PicTar (http: //pictar.mdc-berlin.de/). As was previously described by Huang et al., the gene ontology (GO) and pathway analyses 
Table 2. Primer sequences used for quantitative real-time reverse transcription PCR.

\begin{tabular}{|l|l|}
\hline Symbol & Primer (5' ${ }^{\prime}$ to $\mathbf{3}^{\prime}$ ) \\
\hline miR-205-3p & GGGATTTCAGTGGAGTGTAGTTC \\
\hline miR-429 & GGTAATACTGTCTGGTAAAAGCGT \\
\hline miR-148a-3p & TCAGTGCACTACAGAACTTGGTAA \\
\hline miR-193b-3p & GCCCTCAAAGTCCCGCTA \\
\hline miR-218-5p & TTGTGCTTGATCTAACCATGTAAA \\
\hline miR-449b-5p & AGGCAGTGTATTGTTAGCTAGCA \\
\hline miR-431-3p & CAGGTCGTCTTGCAGGGCTA \\
\hline miR-214-3p & ACAGCAGGCACAGACAGGA \\
\hline miR-133b & GTCCCCTTCAACCAGCTAAA \\
\hline U6 & TTCGTGAAGCGTTCCATATTTT \\
\hline
\end{tabular}

were conducted to annotate the functions of predicted miRNA targets using an online functional annotation tool (The Database for Annotation, Visualization and Integrated Discovery [DAVID] http://david.abcc.ncifcrf.gov/). GO terms and pathways with a p-value $<0.05$ and a false discovery rate $<0.05$ were retained. The Cytoscape software was used to visualize and interpret the relationships between the GO, pathway and predicted target genes of interest for miRNA.

\section{Statistical analysis}

Statistical analyses were performed using the SPSS software for Windows (version 16.0; SPSS Inc., IL, USA). A two-tailed Student's t-test was used to identify miRNAs that were differentially expressed between the SNIP and control groups. Pearson correlation analysis was performed to investigate the linear relationship between the microarray data and qRT-PCR results. Receiver operating characteristic (ROC) curve was established to evaluate diagnostic accuracy of miRNAs as biomarkers of SNIP. Fisher's exact test was used to determine the relationships between clinicopathological characteristics and altered miRNA expression. $\mathrm{p}<0.05$ was considered to indicate a statistically significant difference.

\section{Results}

MiRNA expression profiling in SNIP tissues

To identify differentially expressed miRNAs potentially involved in SNIP, we firstly examined the miRNAs expression profiles using the miRNA microarray technology and detected a total of 58 miRNAs that were significantly differentially expressed in SNIP tissues compared with the corresponding nontumorous tissues from the control group (fold change $>3$; $\mathrm{p}<0.01$ ). Of the 58 miRNAs, 37 were upregulated and 21 were downregulated in the SNIP group. Notably, miR-205-3p and miR-133a-3p were the most remarkably upregulated and downregulated miRNAs, respectively (Figure $1 \&$ Table 3 ).

\section{Validation of the candidate miRNAs by qRT-PCR}

To confirm the microarray data, we performed qRT-PCR for nine candidate miRNAs randomly selected from the 58 differentially expressed miRNAs detected by the microarray experiment. A qRT-PCR was performed in two extend panel of the SNIP group $(n=32)$ and control group $(n=12)$. The qRT-PCR results for seven miRNAs (miR205a-3p, miR-449b-5p, miR-429, miR-133b, miR-193b-3p, miR-148a-3p and miR-214-3p) were consistent with those from the microarray study, while the results for miR-431-3p and miR-218-5p were inconsistent, resulting in a concordance rate of $77.8 \%(7 / 9)$ (Figure 2A). Pearson correlation analysis further indicated a positive correlation between the microarray data and $q$ RT-PCR results $(\mathrm{r}=0.7246, \mathrm{p}=0.0219)$ (Figure 2B).

\section{Enrichment analysis of the potential targets of significantly upregulated and downregulated} miRNAs

To explore the potential functions of the differentially expressed miRNAs in the progression of SNIP, we used three online databases (TargetScan, miRanda, and PicTar) to predict the target genes potentially regulated by these miRNAs. The functional enrichment (GO and pathway annotations) of these miRNA-regulated putative target genes were then analyzed. With GO analysis, we detected several significantly over-represented GO terms involved 


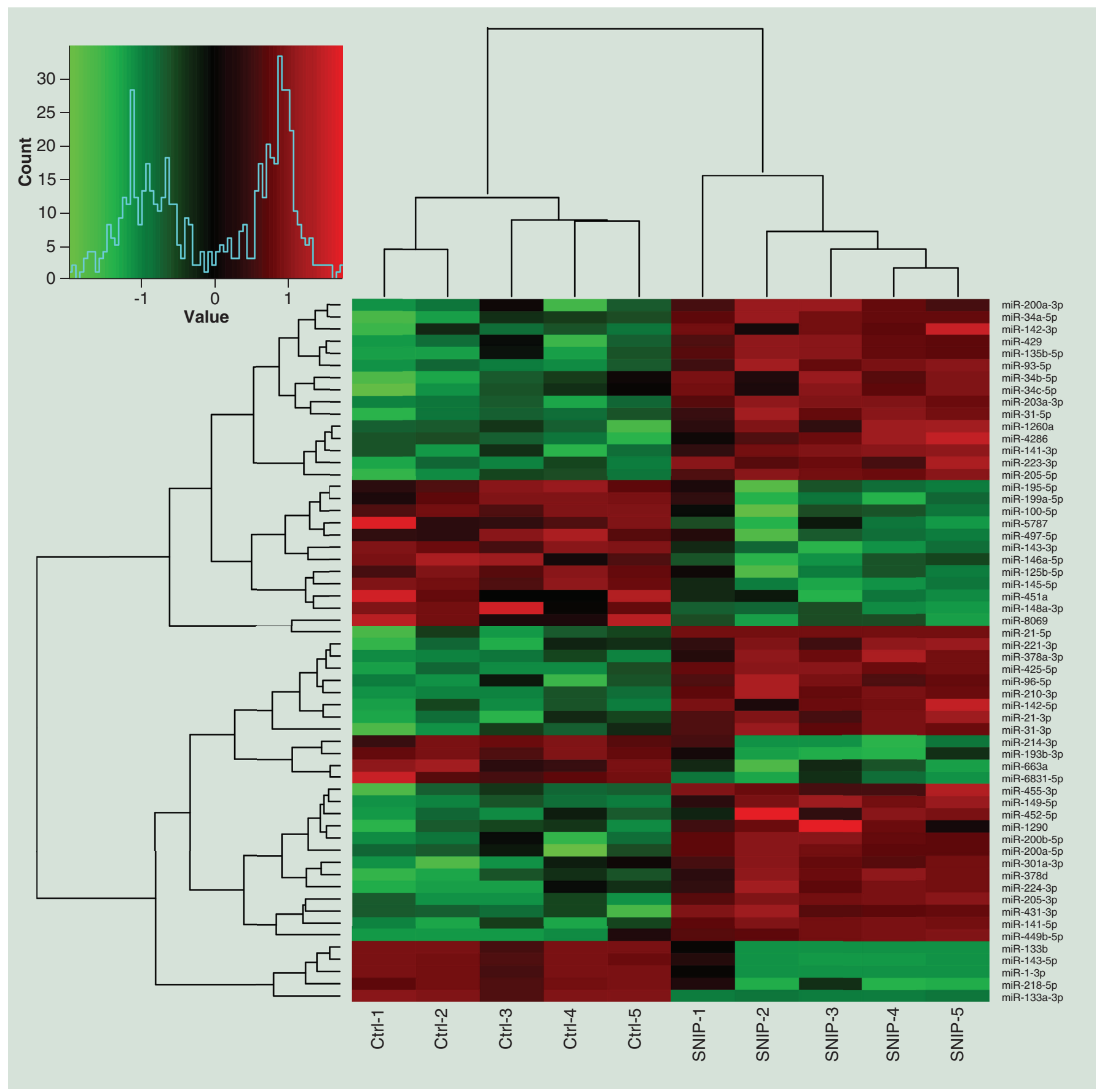

Figure 1. Hierarchical clustering of miRNA expression profiles. A hierarchical cluster analysis was performed with 58 differentially expressed miRNAs as compared the SNIP tissues from the SNIP group $(n=5)$ with nontumorous tissues from the control group ( $n=5$; fold change $>3 ; p<0.01$ ). Each row indicates one miRNA, and each column indicates one sample. The miRNA expression levels are illustrated using a color key and histogram. The red and green bars denote high and low expression, respectively.

in biological process, molecular function and cellular component $(\mathrm{p}<0.05$; false discovery rate $<0.05)$, including cell proliferation and apoptosis, such as positive regulation of cell proliferation and negative regulation of apoptotic process (Figure 3A-C). Pathway analysis showed that a total of 36 significantly enriched pathways $(\mathrm{p}<0.05$; false discovery rate $<0.05$ ). Many of these pathways were associated with the pathogenesis of tumors, such as Wnt signaling pathway, Rat sarcoma (Ras) signaling pathway and PI3K-Akt signaling pathway (Figure 3D). 
Table 3. List of differentially expressed miRNAs in sinonasal inverted papilloma tissues detected by miRNA microarray

\section{assay.}

\begin{tabular}{|c|c|c|c|c|c|c|c|}
\hline \multicolumn{4}{|c|}{ Upregulated miRNAs } & \multicolumn{4}{|c|}{ Downregulated miRNAs } \\
\hline miRNA & Accession no. & $\log _{2}$ fold change & p-value & miRNA & Accession no. & $\log _{2}$ fold change & p-value \\
\hline miR-205-3p & MIMAT0009197 & 7.026609 & $1.51 \mathrm{E}-05$ & miR-133a-3p & MIMAT0000427 & -6.3399 & $1.61 \mathrm{E}-05$ \\
\hline miR-203a-3p & MIMAT0000264 & 6.336157 & $1.34 \mathrm{E}-06$ & $\operatorname{miR}-1-3 p$ & MIMAT0000416 & -6.33986 & 0.000979 \\
\hline miR-31-3p & MIMAT0004504 & 5.752372 & 0.001156 & miR-133b & MIMAT0000770 & -5.92231 & 0.000851 \\
\hline $\operatorname{miR}-141-5 p$ & MIMAT0004598 & 5.217086 & 0.000351 & miR-143-5p & MIMAT0004599 & -4.57649 & 0.003366 \\
\hline $\operatorname{miR}-449 b-5 p$ & MIMAT0003327 & 4.906202 & 0.002795 & $\operatorname{miR}-218-5 p$ & MIMAT0000275 & -4.26118 & 0.007145 \\
\hline miR-31-5p & MIMAT0000089 & 4.878043 & $1.23 \mathrm{E}-05$ & $\operatorname{miR}-145-5 p$ & MIMAT0000437 & -4.05961 & 5.57E-05 \\
\hline miR-431-3p & MIMAT0004757 & 4.550118 & 0.000205 & miR-143-3p & MIMAT0000435 & -3.85758 & 0.000158 \\
\hline $\operatorname{miR}-224-3 p$ & MIMAT0009198 & 4.350782 & 0.001101 & miR-146a-5p & MIMAT0000449 & -3.33842 & 0.000261 \\
\hline $\operatorname{miR}-205-5 p$ & MIMAT0000266 & 3.664981 & 0.000119 & $\operatorname{miR}-451 a$ & MIMAT0001631 & -3.01357 & 0.006868 \\
\hline miR-34c-5p & MIMAT0000686 & 3.465611 & 0.005837 & miR-125b-5p & MIMAT0000423 & -2.77412 & 0.003688 \\
\hline $\operatorname{miR}-210-3 p$ & MIMAT0000267 & 3.42754 & $1.20 \mathrm{E}-06$ & miR-100-5p & MIMAT0000098 & -2.73567 & 0.004155 \\
\hline $\operatorname{miR}-223-3 p$ & MIMAT0000280 & 3.220448 & $8.71 \mathrm{E}-06$ & miR-193b-3p & MIMAT0002819 & -2.1396 & 0.004684 \\
\hline miR-34b-5p & MIMAT0000685 & 3.174226 & 0.00418 & miR-148a-3p & MIMAT0000243 & -2.05114 & 0.000909 \\
\hline miR-378d & MIMAT0018926 & 2.990037 & 0.000275 & miR-195-5p & MIMAT0000461 & -1.86057 & 0.004959 \\
\hline miR-135b-5p & MIMAT0000758 & 2.70132 & 0.00071 & miR-214-3p & MIMAT0000271 & -1.83757 & 0.009267 \\
\hline miR-21-3p & MIMAT0004494 & 2.632962 & 0.000378 & miR-8069 & MIMAT0030996 & -1.77007 & 0.001181 \\
\hline miR-200a-5p & MIMAT0001620 & 2.592559 & 0.004836 & miR-663a & MIMAT0003326 & -1.76273 & 0.001223 \\
\hline miR-96-5p & MIMAT0000095 & 2.563414 & 0.000441 & miR-497-5p & MIMAT0002820 & -1.74511 & 0.005241 \\
\hline $\operatorname{miR}-141-3 p$ & MIMAT0000432 & 2.355117 & 0.000109 & miR-199a-5p & MIMAT0000231 & -1.70426 & 0.005371 \\
\hline miR-301a-3p & MIMAT0000688 & 2.255996 & 0.006952 & miR-6831-5p & MIMAT0027562 & -1.689 & $6.40 \mathrm{E}-05$ \\
\hline miR-429 & MIMAT0001536 & 2.220221 & 0.001187 & miR-5787 & MIMAT0023252 & -1.64863 & 0.000945 \\
\hline miR-149-5p & MIMAT0000450 & 2.10217 & $3.42 \mathrm{E}-05$ & & & & \\
\hline $\operatorname{miR}-452-5 p$ & MIMAT0001635 & 2.093135 & 0.005571 & & & & \\
\hline miR-378a-3p & MIMAT0000732 & 2.087512 & $7.69 \mathrm{E}-05$ & & & & \\
\hline miR-4286 & MIMAT0016916 & 2.039933 & 0.000486 & & & & \\
\hline$m i R-21-5 p$ & MIMAT0000076 & 2.028528 & 0.001311 & & & & \\
\hline miR-200b-5p & MIMAT0004571 & 2.00426 & 0.000976 & & & & \\
\hline miR-142-5p & MIMAT0000433 & 1.845882 & 0.000168 & & & & \\
\hline miR-93-5p & MIMAT0000093 & 1.826103 & $6.67 \mathrm{E}-06$ & & & & \\
\hline $\operatorname{miR}-1290$ & MIMAT0005880 & 1.772368 & 0.001236 & & & & \\
\hline $\operatorname{miR}-425-5 p$ & MIMAT0003393 & 1.762644 & $5.67 \mathrm{E}-06$ & & & & \\
\hline miR-455-3p & MIMAT0004784 & 1.729404 & 0.000237 & & & & \\
\hline miR-200a-3p & MIMAT0000682 & 1.69094 & 0.001973 & & & & \\
\hline $\operatorname{miR}-221-3 p$ & MIMAT0000278 & 1.679623 & 0.000828 & & & & \\
\hline miR-142-3p & MIMAT0000434 & 1.676354 & 0.000414 & & & & \\
\hline miR-1260a & MIMAT0005911 & 1.612317 & 0.000335 & & & & \\
\hline $\operatorname{miR}-34 a-5 p$ & MIMAT0000255 & 1.598804 & 0.001324 & & & & \\
\hline
\end{tabular}

Evaluation of miR-214-3p as a potential biomarker of SNIP

To verify whether miR-214-3p could discriminate the SNIP patients from controls, we performed qRT-PCR in two extended panel of the SNIP group $(n=32)$ and the control group $(n=12)$, and established ROC curve to determine its specificity and sensitivity as a diagnostic biomarker. ROC curve analysis revealed that miR-214-3p had a high accuracy in differentiating the SNIP patients from controls: area under the curve $=0.932$; $95 \%$ CI: $0.834-1.031$; sensitivity $=96.9 \%$, specificity $=83.3 \%$ (Figure 4 ). 


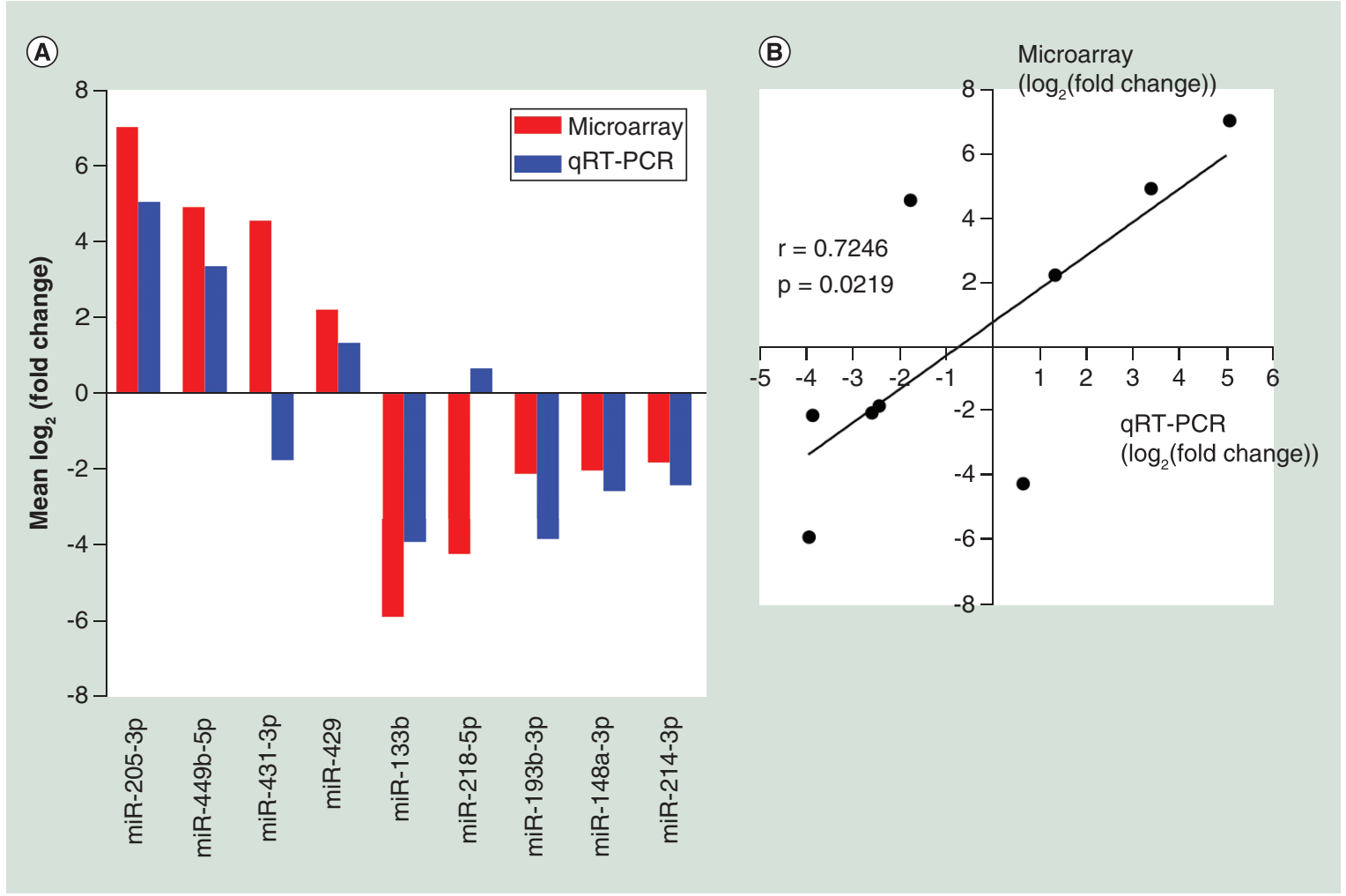

Figure 2. The qRT-PCR validation of nine differentially expressed miRNAs selected by the microarray assay. (A) Comparison of miRNA expression levels obtainedby miRNA microarray and qRT-PCR analysis. Upregulated and downregulated miRNAs are indicated by bars above and below the horizontal axis, respectively. (B) Pearson correlation analysis investigating the linear relationship between the microarray data and qRT-PCR results. qRT-PCR: Quantitative real-time reverse transcription PCR.

Construction of a network reflecting the relationships between miR-214-3p, its predicated targets and associated GO \& pathway terms

To further investigate the biological functions of significantly downregulated miR-214-3p in SNIP tissues, we constructed a network of miR-214-3P, its predicted targets and associated GO and pathway terms. As shown in Figure 5, many genes, such as CDKG, BCL2L11, KPNA3 and ITCH, could be considered as the potential targets of miR-214-3p. These target genes were enriched in several GO and pathway terms linked to virus infection and pathogenesis of tumors, such as modulation by virus of host morphology or physiology, viral entry into host cell, p53 signaling pathway and Notch signaling pathway.

\section{Relationships between clinicopathological characteristics \& miR-214-3p expression}

To determine the potential clinicopathological implications of the altered miR-214-3p expression, the real-time PCR results and clinicopathological characteristics from 32 patients with SNIP were analyzed using Fisher's exact test. Clinicopathologic analysis revealed that the downregulation of miR-214-3p in SNIP was significantly related to tumor staging $(\mathrm{p}=0.021)$ and recurrence $(\mathrm{p}=0.038)$ (Table 4$)$.

\section{Discussion}

Although SNIP is known to be a locally destructive, benign neoplasm of the nose and paranasal sinuses with a high tendency for recurrence and the possibility of malignant transformation, the underlying molecular mechanisms are still largely unknown. $p 53, D L E C 1$, gelsolin and caspase-3 have been reported to be abnormally expressed in SNIP tissue, some of which are possibly associated with the occurrence and development of SNIP [15-17]. However, it has yet to be clarified whether and how the expression of these genes is fine-tuned at the post-transcriptional level. Such information will contribute to the development of preventative and therapeutic strategies against SNIP. 
Table 4. Relationships between clinicopathological characteristics and miR-214-3p expression in patients with sinonasal

\section{inverted papilloma.}

\begin{tabular}{|c|c|c|c|}
\hline \multirow[t]{2}{*}{ Parameter } & \multicolumn{3}{|c|}{ miR-214-3p } \\
\hline & Upregulation $^{\dagger}(\%), \mathrm{N}=17$ & Downregulation $\ddagger$ (\%), $\mathrm{N}=15$ & p-value (Fisher's exact test) \\
\hline Age: & & & 1.000 \\
\hline$-<50$ years & $6(35.3)$ & $5(33.3)$ & \\
\hline$-\geq 50$ years & $11(64.7)$ & $10(66.7)$ & \\
\hline Gender: & & & 0.383 \\
\hline - Male & $15(88.2)$ & $11(73.3)$ & \\
\hline - Female & $2(11.8)$ & $4(26.7)$ & \\
\hline Smoking status: & & & 0.712 \\
\hline - Yes & $12(70.6)$ & $9(60.0)$ & \\
\hline$-\mathrm{No}$ & $5(29.4)$ & $6(40.0)$ & \\
\hline Tumor staging: $\S$ & & & 0.021 \\
\hline$-1+I I$ & $15(88.2)$ & $7(46.7)$ & \\
\hline$-I I I+I V$ & $2(11.8)$ & $8(53.3)$ & \\
\hline Recurrence: & & & 0.038 \\
\hline - Yes & $0(0.0)$ & $4(26.7)$ & \\
\hline$-\mathrm{No}$ & $17(100.0)$ & $11(73.3)$ & \\
\hline $\begin{array}{l}\dagger \text { Greater than or ec } \\
\ddagger \text { Less than } 0.17 \text {. } \\
\text { §Tumor staging is }\end{array}$ & $\begin{array}{l}\text { ve expression level of miR-214- } \\
\text { ing system for inverted papillon }\end{array}$ & $\begin{array}{l}\text { ts with sinonasal inverted papillor } \\
\text { Krouse (2000). }\end{array}$ & \\
\hline
\end{tabular}

MiRNAs are a highly conserved class of short, noncoding RNAs that function as important post-transcriptional regulators, playing vital roles not only in regulating individual growth and development but also in tuning the

(A)

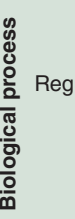
Regulation of transcription DNA-dependent Negative regulation of cell proliferation Immune response

Positive regulation of transcription DNA-dependent Epidermal growth factor recription DNA-dependent Nerve growth factor receptor signaling pathway Nerve growth factor receptor signaling pathway Small GTPase mediated signal transduction Actin cytoskeleton organization Signal transduction
Viral transcription Viral transcription phosphorylation Transcription from RNA polymerase II promoter Fibroblast growth factor receptor signaling pathway rRNA processing Virus-host interaction Protein autophosphorylation Wnt receptor signaling pathway Transforming growth factor beta receptor signaling pathway Negative regulation of endopeptidase activity Protein ubiquitination

Ubiquitin-dependent protein catabolic process Proteasomal ubiquitin-dependent protein catabolic process Negative regulation of apoptotic process Positive regulation of cell migration Positive regulation of cell migration
Positive regulation of GTPase activity Positive regulation of GTPase activity
Positive regulation of cell proliferation Positive regulation of cell proliferation Protein K48-linked ubiquitination M/G1 transition of mitotic cell cycle

\begin{tabular}{ccc}
$-\lg (\mathbf{p}$-value) \\
5 & 10 & 15 \\
1 & 1 & \\
\hline & & \\
\hline & \\
\hline
\end{tabular}

(B)

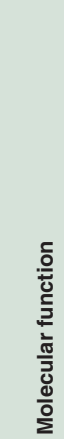

B) $\quad-\lg (p$-value)

G-protein coupled receptor activity Structural constituent of ribosome

Sequence-specific DNA binding

Ubiquitin-protein ligase activity Protein kinase binding Zinc ion binding Chromatin binding Protein serine/threonine kinase activity Transcription factor binding Beta-catenin binding DNA binding SMAD binding Serine-type endopeptidase inhibitor activity Protein domain specific binding Transcription regulatory region DNA binding

Transcription coactivator activity

Transcription corepressor activity Signal transducer activity Electron carrier activity Protein transporter activity Actin binding Calmodulin binding RNA binding
Ubiquitin protein ligase binding PDZ domain binding -

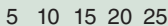

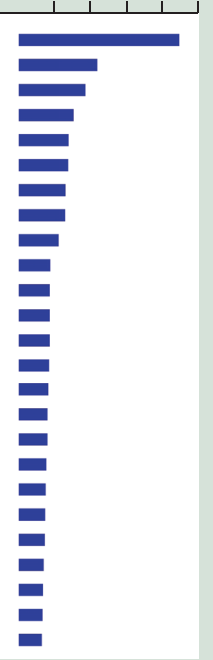

Figure 3. An enrichment analysis of the predicted targets of 58 differentially expressed miRNAs in the SNIP tissues. (A-C) GO functional enrichment of miRNA potential targets: (A) biological process, (B) molecular fuction, (C) cellular component. (D) Pathway annotation of the target genes of the miRNAs. Ordinate is the significant GO/pathway term $(p<0.05, F D R<0.05)$. 


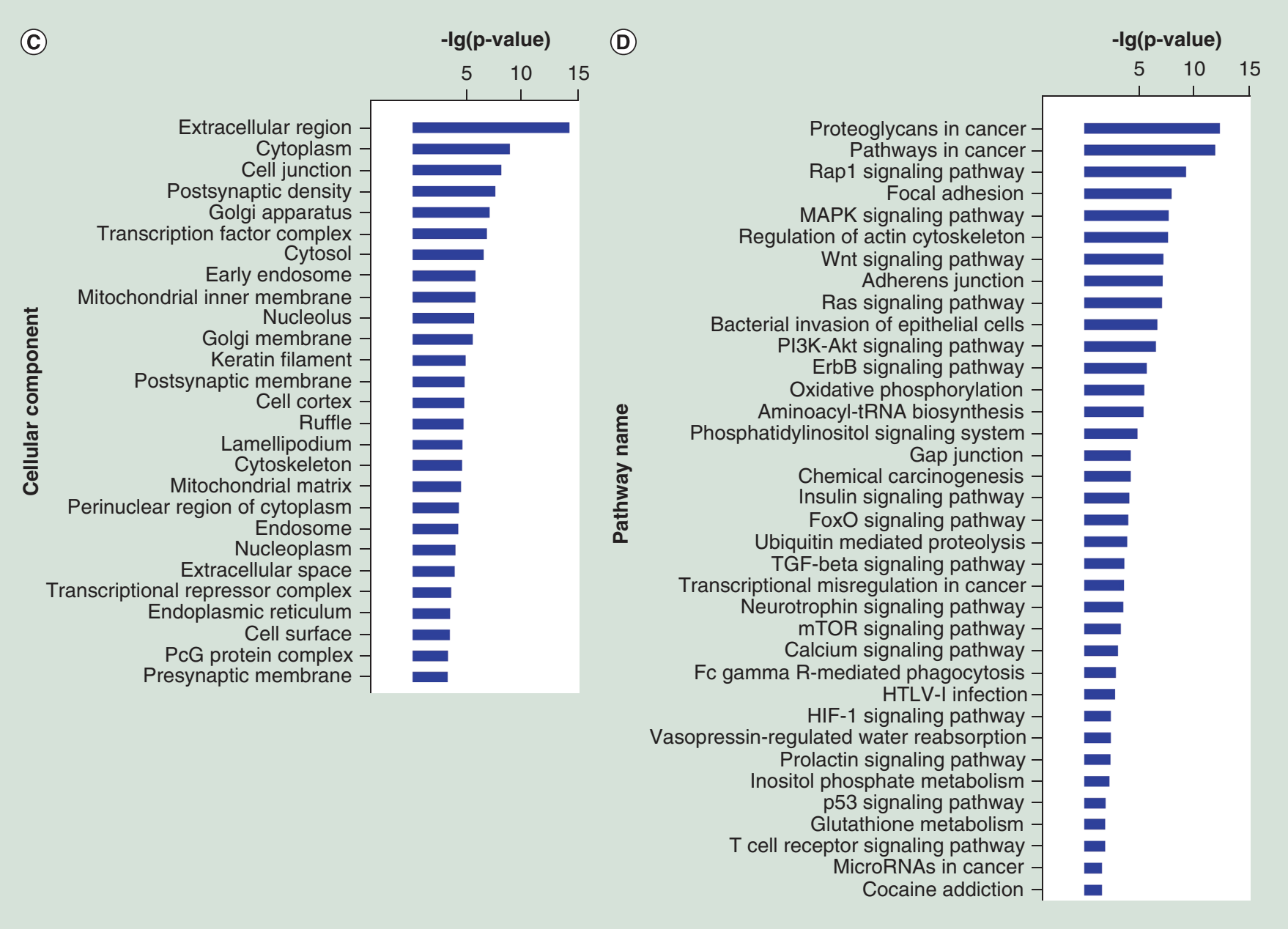

Figure 3. An enrichment analysis of the predicted targets of 58 differentially expressed miRNAs in the SNIP tissues (cont.). (A-C) GO functional enrichment of miRNA potential targets: (A) biological process, (B) molecular fuction, (C) cellular component. (D) Pathway annotation of the target genes of the miRNAs. Ordinate is the significant GO/pathway term $(p<0.05, F D R<0.05)$.

Figure 4. ROC curve analysis used to assess on the accuracy of miR-214-3p in discriminating the SNIP patients.

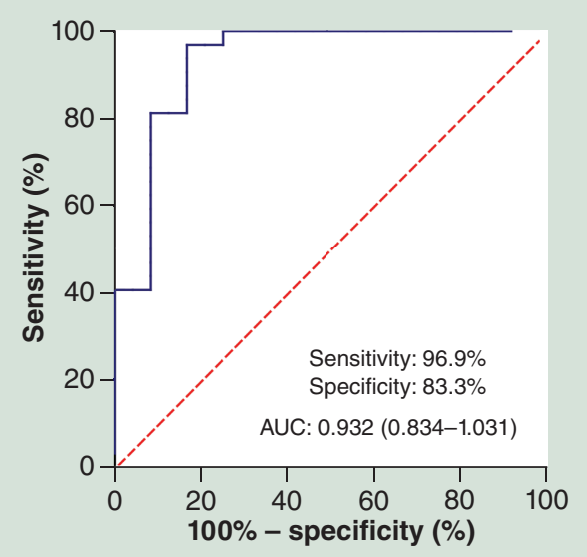




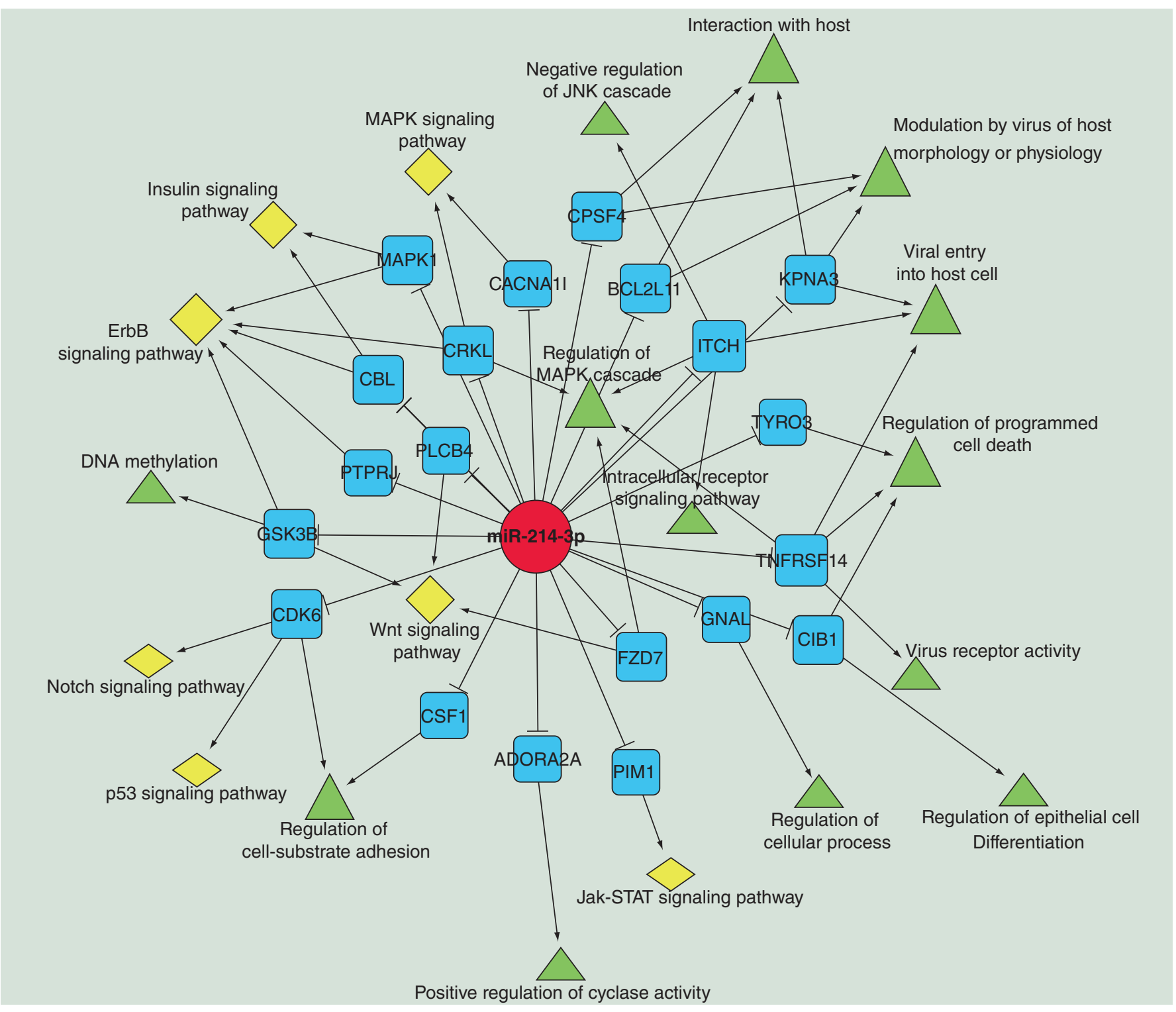

Figure 5. Predicted targets of miR-214-3p and their annotated GO and pathway terms. miR-214-3p is indicated by a red circle. Its target genes are indicated by blue squares. GO and pathway terms are represented by green triangles and yellow diamonds, respectively.

expression of well-known genes involved in tumorigenesis [9,18]. Emerging evidence has indicated that several aberrantly expressed miRNAs are profoundly involved in the pathogenesis of head and neck neoplasms [19]. As reported by Kakizaki et al. [14], miR-296-3p was markedly upregulated in squamous cell carcinoma and might play a critical role in the malignant transformation of SNIP via the regulation of PTEN and the subsequent inhibition of the PI3K/Akt signaling pathway. To further elucidate the roles of miRNAs in the pathogenesis of SNIP, we studied the miRNA profiles in SNIP tissue using a microarray-based screening method and detected 58 candidate miRNAs (37 upregulated and 21 downregulated miRNAs) that had a threefold or greater change in expression. Nine of these miRNAs were then analyzed by qRT-PCR to confirm the reliability of the microarray data. We observed a high concordance rate of $77.8 \%$ and a positive correlation between the microarray data and qRT-PCR results.

The biological function of miRNAs is achieved mainly by manipulating the stability of mRNA and/or the initiation and progression of protein translation. Thus, the identification of miRNA target genes is considered to be a crucial step toward clarifying the miRNAs function. In our study, we intersected three prediction databases (TargetScan, miRanda and PicTar) in order to identify potential target genes of the differentially expressed miRNAs in SNIP tissues. These target genes can be classified into several functional categories by GO and pathway analyses 
based on the DAVID tools. DAVID is composed of an integrated biological knowledge base and analytic tools to understand biological meaning from large gene/protein lists. The procedure first requires uploading a gene list containing any number of common gene identifiers followed by analysis using one or more text and pathway-mining tools such as gene functional classification, functional annotation clustering, chart or table. Finally, investigators are able to obtain an in-depth understanding of the biological themes in lists of genes that are enriched in genome-scale studies. Interestingly, by following this protocol, we detected several genes that are involved in multiple tumorrelated biological processes and signaling pathways, such as positive regulation of cell proliferation, Wnt signaling pathway and Ras signaling pathway. These findings suggest that the differentially expressed miRNAs detected in this study might participate in the pathogenic process of SNIP by regulating their target gene expression.

Recently, a growing number of studies have indicated the potential of using miRNAs as biomarkers in disease diagnoses and prognosis [20-22]. For example, serum miR-378 has been suggested to be a noninvasive diagnostic biomarker for early detection of gastric cancer [23]. Kovarikova et al. [24] reported significantly upregulated miR-21 in sinonasal cancer tissue and sinonasal carcinoma patients and impaired survival of the patients with a high expression level of miR-21, suggesting that miR-21 could act as a valuable prognostic biomarker. Along with these clues, we investigated which of the differently expressed miRNAs in SNIP tissue could be used as diagnostic biomarkers. Our study showed that miR-214-3p could yield a ROC curve area of 0.932 with $96.9 \%$ sensitivity and $83.3 \%$ specificity in discriminating SNIP patients from controls. Thus, miR-214-3p probably provides great potential as a novel biomarker in molecular pathological diagnosis of SNIP. However, its specificity needs to be tested in depth between the SNIP and other nasal tumors. In addition, it remains to be further studied whether plasma/serum miR-214-3p can serve as a noninvasive diagnostic biomarker for early detection of SNIP.

As is well known, HPV infection is a definite risk factor for SNIP and plays an important role in the clinical evolution of SNIP [25-27]. However, it is not yet clear whether miRNAs are involved in the pathogenesis of HPVinduced SNIP. Sannigrahi et al. [28] demonstrated that HPV-16-mediated downregulation of miR-139-3p might promote oncogenesis in head and neck cancer by miR-139-3p targeting high-risk HPV-16 oncogenic proteins and revive major tumor suppressor proteins (p53, p21 and p16). Our bioinformatics data revealed that some of the putative target genes of miR-214-3p are related to the biological processes and mechanisms of virus infection, such as virus of host morphology or physiology and viral entry into host cells. Their relationships were visualized by the Cytoscape software, which is an open source software platform for visualizing molecular interaction networks and biological pathways and integrating these networks with annotations, gene expression profiles and other state data. In the working window of the Cytoscape software, you can import data files, such as expression profiles or GO annotations, generated by other applications or spreadsheet programs. These data can be mapped to node color, label, border thickness or border color, etc. according to user-configurable colors and visualization schemes.

In addition, we found that the characteristic expression of miR-214-3p was significantly associated with tumor staging and recurrence of SNIP. Given that SNIP is characterized by local invasiveness, tendency to recurrence, it is very necessary to detect the expression level of miR-214-3p in tissue samples from each patient with SNIP, which will contribute to evaluating the prognosis of SNIP patients. Therefore, our findings provided a possibility that miR-214-3p may serve as a gene expression regulator involved in HPV infection and oncogenesis of SNIP.

\section{Conclusion}

Our investigation, although preliminary, has revealed the potential biological functions of miRNAs in SNIP, providing new insight into the related molecular mechanism. We have further reported the strong correlation between the downregulation of miR-214-3p and tumor staging and recurrence of SNIP and identified miR-214-3p as a promising biomarker in SNIP. Such information has important clinical implications and would be helpful in identifying novel therapeutic targets for the treatment of SNIP patients.

\section{Author's contributions}

YS Teng, ZH Lin and Y Li conceived and designed the experiments. ZH Lin, YQ Gao and XL Cao have made substantial contribution to collected tissue samples. YS Teng, FC Lin, XY Lou and YD Li performed the experiments and analyzed the data. YS Teng and YD Li prepared the figures and tables and drafted the manuscript. All authors read and approved the final manuscript.

Financial \& competing interests disclosure

This work was supported by grants from Medical and Health Technology Program of Zhejiang (No: 2013RCA041), Science and Technology Development Project of Hangzhou (No: 20160533B09), Medical and Health Technology Program of Hangzhou (No: 
2014A08), and Zhejiang Provincial Natural Science Foundation of China (No: LY17H130001). The authors have no other relevant affiliations or financial involvement with any organization or entity with a financial interest in or financial conflict with the subject matter or materials discussed in the manuscript apart from those disclosed.

No writing assistance was utilized in the production of this manuscript.

Ethical disclosure

This study complies with the Declaration of Helsinki and was approved by the Local Ethics Committee. In addition, for investigations involving human subjects, informed consent has been obtained from the participants involved.

Open access

This work is licensed under the Attribution-NonCommercial-NoDerivatives 4.0 Unported License. To view a copy of this license, visit http://creativecommons.org/licenses/by-nc-nd/4.0/

\section{Summary points}

Background

- Sinonasal inverted papilloma (SNIP) is a histologically benign lesion. However, it is characterized by local invasiveness, a high recurrence rate and a tendency for malignant transformation.

- Little is known about the underlying molecular genetic alterations and physiopathologic mechanism of this clinical entity.

- Dysregulation of miRNAs has been implied in the progression of tumorigenesis. However, their role and clinicopathological significance in SNIP have yet to be identified.

MiRNA expression profiling in SNIP tissues

- The miRNA microarray assay identified a total of 58 significantly expressed (37 upregulated and 21 downregulated) miRNAs in SNIP tissues.

- The potential target genes of these significantly expressed miRNAs were involved in multiple tumor-related biological processes and signaling pathways, such as positive regulation of cell proliferation, Wnt signaling pathway, and Ras signaling pathway.

MiR-214-3p as a novel biomarker in SNIP

- MiR-214-3p had high accuracy in differentiating the SNIP patients from controls: area under the curve =0.932; $95 \% \mathrm{Cl}$ : 0.834-1.031; sensitivity $=96.9 \%$, specificity $=83.3 \%$.

- Downregulation of miR-214-3p in SNIP was significantly related to tumor staging $(p=0.021)$ and recurrence $(p=$ 0.038).

Conclusion

- MiRNAs might participate in the pathogenic process of SNIP by regulating their target gene expression.

- Our data might offer new ideas that miR-214-3p probably serves as a valuable biomarker and a therapeutic target for SNIP.

\section{References}

Papers of special note have been highlighted as: $\bullet$ of interest; $\bullet \bullet$ of considerable interest

1. Vrabec DP. The inverted Schneiderian papilloma: a 25-year study. Laryngoscope 104(5 Pt 1), 582-605 (1994).

2. Lawson W, Ho BT, Shaari CM, Biller HF. Inverted papilloma: a report of 112 cases. Laryngoscope 105(3 Pt 1), 282-288 (1995).

3. Lawson W, Patel ZM. The evolution of management for inverted papilloma: an analysis of 200 cases. Otolaryngol. Head Neck Surg. 140(3), 330-335 (2009).

4. Krouse JH. Endoscopic treatment of inverted papilloma: safety and efficacy. Am. J. Otolaryngol. 22(2), 87-99 (2001).

5. Govindaraj S, Wang H. Does human papilloma virus play a role in sinonasal inverted papilloma? Curr. Opin. Otolaryngol. 22(1), 47-51 (2014).

6. Ambros V. The functions of animal microRNAs. Nature 431(7006), 350-355 (2004).

7. Bartel DP. MicroRNAs: genomics, biogenesis, mechanism, and function. Cell 116(2), 281-297 (2004).

- A review article highlighting what has been learned about miRNAs since the report of the lin- 4 RNA. The major topics discussed were miRNA genomics, miRNA biogenesis, miRNA regulatory mechanisms and the roles of miRNAs in gene regulatory pathways.

8. Nannini M, Ravegnini G, Angelini S et al. miRNA profiling in gastrointestinal stromal tumors: implication as diagnostic and prognostic markers. Epigenomics 7(6), 1033-1049 (2015).

9. Jansson MD, Lund AH. MicroRNA and cancer. Mol. Oncol. 6(6), 590-610 (2012). 
10. Mattes J, Collison A, Foster PS. Emerging role of microRNAs in disease pathogenesis and strategies for therapeutic modulation. Curr. Opin. Mol. Ther. 10(2), 150-157 (2008).

11. Shen Z, Qin X, Yan M et al. Cancer-associated fibroblasts promote cancer cell growth through a miR-7-RASSF2-PAR-4 axis in the tumor microenvironment. Oncotarget 8(1), 1290-1303 (2017).

12. Obayashi M, Yoshida M, Tsunematsu T et al. microRNA-203 suppresses invasion and epithelial-mesenchymal transition induction via targeting NUAK1 in head and neck cancer. Oncotarget 7(7), 8223-8239 (2016).

- A research article identifying miRNA expression profiles between a parent head and neck squamous cell carcinoma (HNSCC) cell line and a highly invasive clone using miRNA microarray. The article also suggested the role and mechanism of a specific miRNA in invasion and epithelial-mesenchymal transition induction of HNSCC cells.

13. Mitra S, Mukherjee N, Das S et al. Anomalous altered expressions of downstream gene-targets in TP53-miRNA pathways in head and neck cancer. Sci. Rep. 4, 6280 (2014).

14. Kakizaki T, Hatakeyama H, Nakamaru Y et al. Role of microRNA-296-3p in the malignant transformation of sinonasal inverted papilloma. Oncol. Lett. 14(1), 987-992 (2017).

-• A research article identifying several differentially expressed miRNAs between sinonasal inverted papilloma (SNIP) and squamous cell carcinoma into which a proportion of SNIPs transform. The article also highlighted that miRNA-296-3p might serve an important role in the malignant transformation of SNIP.

15. Altavilla G, Staffieri A, Busatto G et al. Expression of p53, p16INK4A, pRb, p21WAF1/CIP1, p27KIP1, cyclin D1, Ki-67 and HPV DNA in sinonasal endophytic Schneiderian (inverted) papilloma. Acta Otolaryngol. 129(11), 1242-1249 (2009).

- A research article describing the relationships between HPV infection and aberrant expression of cell cycle proteins in SNIP.

16. Chang PH, Huang CC, Lee TJ et al. Downregulation of DLEC1 in sinonasal inverted papilloma and squamous cell carcinoma. J. Otolaryngol. Head Neck Surg. 41(2), 94-101 (2012).

17. Cho JE, Park W, Kim DC et al. Down-regulation of gelsolin may play a role in the progression of inverted papilloma through an antiapoptotic mechanism. Am. J. Rhinol. Allergy 26(3), 177-182 (2012).

18. Nohata N, Hanazawa T, Kinoshita $\mathrm{T}$ et al. MicroRNAs function as tumor suppressors or oncogenes: aberrant expression of microRNAs in head and neck squamous cell carcinoma. Auris Nasus Larynx 40 (2), 143-149 (2012).

- A review article evaluating the aberrant expression of miRNAs by analyzing eight genome-wide miRNA expression profiles of HNSCC and their contribution to human HNSCC oncogenesis.

19. Koshizuka K, Hanazawa T, Arai T et al. Involvement of aberrantly expressed microRNAs in the pathogenesis of head and neck squamous cell carcinoma. Cancer Metastasis Rev. 36(3), 525-545 (2107).

20. Shekari N, Baradaran B, Shanehbandi D et al. Circulating microRNAs: valuable biomarkers for diagnosis and prognosis of gastric cancer. Curr. Med. Chem. 25(6), 698-714 (2108).

21. Yang $Y, H u Z$, Zhou $Y$ et al. The clinical use of circulating microRNAs as non-invasive diagnostic biomarkers for lung cancers. Oncotarget 8(52), 90197-90214 (2017).

- A review article showing a comprehensive evaluation of diagnostic role of circulating miRNAs in lung cancer using meta-analysis. It provided an important clue that circulating miRNAs, particularly the combination of multiple miRNAs, might serve as promising biomarkers for the diagnosis of human diseases.

22. Ji X, Takahashi R, Hiura Y et al. Plasma miR-208 as a biomarker of myocardial injury. Clin. Chem. 55(11), 1944-1949 (2009).

23. Liu H, Zhu L, Liu B et al. Genome-wide microRNA profiles identify miR-378 as a serum biomarker for early detection of gastric cancer. Cancer Lett. 316(2), 196-203 (2012).

24. Kovarikova H, Bubancova I, Laco J et al. Deregulation of selected microRNAs in sinonasal carcinoma: value of miR-21 as prognostic biomarker in sinonasal squamous cell carcinoma. Head Neck 39(12), 2528-2536 (2017).

-. A research article describing miR-21 as a prognostic biomarker in sinonasal squamous cell carcinoma by Kaplan-Meier analysis with log-rank survival.

25. Lin H, Lin D, Xiong X. Roles of HPV infection and Stathmin in the pathogenesis of sinonasal inverted papilloma. Head Neck 38(2), 220-224 (2014).

26. Giotakis E, Gomatos IP, Alevizos L et al. Apoptotic and proliferative status in HPV (+) and HPV (-) inverted papilloma patients. Correlation with local recurrence and clinicopathological variables. Pathol. Res. Pract. 208(6), 338-343 (2016).

27. Scheel A, Lin GC, McHugh JB et al. Human papillomavirus infection and biomarkers in sinonasal inverted papillomas: clinical significance and molecular mechanisms. Int. Forum Allergy Rh. 5(8), 701-707 (2015).

28. Sannigrahi MK, Sharma R, Singh V et al. Role of host miRNA Hsa-miR-139-3p in HPV-16-induced carcinomas. Clin. Cancer Res. 23(14), 3884-3895 (2017).

- A research article describing the role of miRNAs in HPV-related tumors, which provide a meaningful clue for our hypothesis. 
